\title{
Sliding Objects with Random Friction
}

\author{
Itzhak Webman, José Luis Gruver, and Shlomo Havlin \\ Department of Physics and Jack and Pearl Resnick Institute of Advanced \\ Technology, Bar-Ilan University, Ramat-Gan 52900, Israel
}

\begin{abstract}
We study the motion of elastic networks driven over a random substrate. Our model which includes local friction forces leads to complex dynamical behavior. We find a transition to a sliding state which belongs to a new universality class. The phase diagram comprises of a pinned state, a stick-slip motion phase, and a free motion phase.
\end{abstract}

\section{Introduction}

The dynamics of elastic objects driven through an environment with random pinning interactions is complex and its present understanding still incomplete. Processes of this type occur in microscopic systems such as charge density waves driven by an electric field [1,2], superconductors subject to external magnetic fields [3], and polymers moving through a inhomogeneous environment. Related processes on a macroscopic scale occur in the motion of geological faults [4-6], or in sliding friction between an elastic object and a rigid one [7]. Theoretical studies of these phenomena are usually modeled by an elastic network moving in a rigid environment or over a rigid substrate with random interactions between them. In the simplified case where one of the two objects is homogeneous, one may distinguish between random network models, where the random interactions are assigned to sites on the elastic network while the medium or substrate are homogeneous, and the random substrate, models where the random interactions are assigned to the sites of the substrate or the embedding medium while the elastic network is homogeneous. These two type of models are illustrated in Fig. 1

Random network models were invoked to describe randomly pinned charge density waves (CDW) in solids [8]-[13] and were widely studied. Most recent work on random substrate models concentrated on the high velocity limit, where the distortions of the network induced by the random interactions are 
mild [14]-[17], while the dynamical properties near the critical external field for depinning received less attention. We focus on the simplest discrete realization of the random substrate model containing no ad-hoc features such as velocity dependent forces [5], and compare it to the analogous random network model. The study shows that the dynamics of the random substrate model above depinning threshold is interesting and complex, featuring several distinct regimes. In contrast, in the random network case the elastic object follows a simple steady state motion. The pinned state of the random substrate model below threshold is characterized by a wide distribution of strain avalanches. Similar avalanches occur in the random network model, however the critical exponents describing the distributions of avalanche sizes are definitely different.

The properties of the discrete random substrate model depend on three parameters: The external field, the ratio of pinning strength to the elastic stiffness, and the characteristic size of the local pinning regions. We find that whereas in some parts of the relevant phase diagram the two models are approximately equivalent, through most of it there are pronounced differences between them, and the critical behavior belongs to distinct universality classes.

\section{The Model}

Sliding elastic objects are represented here by a discrete chain of particles connected by springs. (see Fig. 1) In the random network limit each particle is attributed a random pinning force, while the substrate is homogeneous. For an external force $E$ switched on at $t=0$ acting uniformly on all particles, the dynamics of the displacements of the positions of the particles from their initial equilibrium positions: $\xi_{i}=x_{i}(t)-x_{i}(0)$ is described by the following overdamped equations of motion:

random network

$$
\partial \xi_{i} / \partial t=\max \left(0, \kappa\left(\xi_{i+1}+\xi_{i-1}-2 \xi_{i}\right)+E-V(i)\right)
$$

This realization of the random network model, also known as the ratchet model [9] successfully describes the dynamical phase transition of charge density waves from a pinned state to a DC current conducting state at a critical external field $E_{c}[9-12,18]$.

In the random substrate model the particles interact with the substrate via local pinning forces $V\left(x_{i}\right)$, whereby the i'th particle at position $x_{i}$ (in the substrate frame of reference) moves if the total force acting on it is greater 
than $V\left(x_{i}\right)$. As before, the dynamics for the displacements: $\xi_{i}=x_{i}-x_{i}(0)$, is described by the following equations:

random substrate

$$
\partial \xi_{i} / \partial t=\max \left(0, \kappa\left(\xi_{i+1}+\xi_{i-1}-2 \xi_{i}\right)+E-V\left(\xi_{i}+i\right)\right)
$$

Initially, the particles positions form a 1D lattice with unit spacing: $x_{i}(0)=i$. Pinning forces are local, with a characteristic range $\Delta$. In our study the pinning force is constant inside lattice unit cells, $(\Delta=1)$, and varies randomly from one cell to the next. We chose a binary distribution for $V$ of the type: $\rho(V)=$ $p \delta\left(V-V_{1}\right)+(1-p) \delta\left(V-V_{0}\right)$, usually with $\mathrm{p}=0.5$. We adopt periodic boundary conditions, so that the argument of $V$ in equation $(1)$ is $\xi_{i} \bmod (L)+i$.

Both models also depict a discrete one dimensional elastic interface moving in the $\xi$ direction in a two dimensional $(i, \xi)$ plane [10], where the interface is initially flat. In the random substrate model a pinning site on the $i$ axis, transforms into lines of pinning sites of slope unity in the $(i, \xi)$ plane, as described in Fig. 2. In the random network case, pinning sites form columns of fixed pinning strength $V_{i}$ in the same plane.

\section{Approximate scaling properties}

The dependence of the general behavior of the random substrate model on the elastic stiffness $\kappa$, and on the size $\Delta$ of regions where pinning forces do not vary, can be simplified by the following scaling relation [19] (provided $\max (\kappa, \kappa \Delta)<1)$ :

$$
\xi(\kappa, \Delta, t)=\kappa^{-1} \xi(1, \kappa \Delta, t / \kappa)
$$

Extending this scaling relation to $\Delta>1$ results in a hybrid random networkrandom substrate model, so that the pure random substrate model is not preserved. However, in the limit $\Delta>>1$ the pure random network model is approached, so that the random network model and the random substrate model approach each other in the very high stiffness limit, as $\kappa \rightarrow \infty$.

\section{Dynamical properties}

In the random network model above the threshold $E_{c}$ all particles eventually move with the same finite velocity as a rigid distorted object. Adding the $N$ equations of motion Eq. (1) the elastic forces sum-up to zero, so that the 
velocity of the center of mass is: [9]

$$
U_{R N}=E-E_{c}
$$

where for a chain of $N$ particles $E_{c}=\frac{1}{N} \sum V_{i}$

The dynamical behavior of the random substrate model is more complex, and two distinct regimes appear as the driving force is varied above the depinning threshold $E_{1}$ :

stick - slip regime : $E_{1}<E<E_{2}$

The center of mass of the system moves with a roughly constant velocity, modulated by fast fluctuations, although at any time a finite fraction of the particles are not in motion.

The center of mass velocity $U$ obtained numerically for several values of stiffness $\kappa$ is shown in Fig. 3. Each curve corresponds to a single representative random configuration for chains $\mathrm{N}=100-1000$. At the threshold there is a small discontinuity, followed by a linear $E$ dependence. The fractions of particles that are in motion at a given instant corresponding to the velocity curves in Fig 3. appear in the inset.

Deriving the CM velocity by summing the individual equations Eq. (2) is more subtle in the random substrate case, since the equations for particles with zero velocity are actually inequalities. Time averages over sufficiently long periods are equivalent to those obtained from a linearized version of Eq. $(1,2)$ where the ratchet condition is omittted, leading to:

$$
U_{R S}=E-\sum n\left(V_{i}, E\right) V_{i}
$$

where $n\left(V_{i}, E\right)$ is the time averaged fraction of particles occupying the i'th unit cell. Since the residence time of particles over a strong pinning area is longer than over a weak pinning one, the mean occupation fractions $n\left(V_{i}, E\right)$ are monotonously increasing functions on $V_{i}$, so that for two systems sharing the same distribution of random pinning strengths, the mean velocity of the random network is always greater than that of the random substrate system, asymptotically approaching it from below in the limit of high velocity. The threshold $E_{1}$ is the solution of the equation:

$$
E_{1}-\sum n\left(V_{i}, E_{1}\right) V_{i}
$$

so that the inequality $E_{1}>E_{c}$ is always valid. 
The center of mass velocity of the random substrate model can be expressed as a driving force minus a velocity dependent drag force $F(U)$ :

$$
U_{R S}=E-F\left(U_{R S}\right)
$$

Analysis of the velocity data shows that $F(U)$ is equal to the static friction force $E_{1}$ in the limit of zero velocity, increases with increasing velocity above $E_{1}$ reaching some plateau, eventually tending to $E_{c}$ for high velocities. This weakening of the kinetic friction force with increasing velocity is generated by a detachment instability where the fraction of immobile particles abruptly vanishes (Fig. 3 inset). Qualitatively similar features were observed in experiments where an elastic membrane is dragged over a rigid substrate, performing stick-slip motion. [7]

The center of mass velocity for $E$ just above the depinning transition scales as $\left(E-E_{1}\right)^{\beta}$. For the random network model, trivially, $\beta=1$. Our results for the random substrate model also follow a linear $E-E_{1}$ dependence, i.e. $\beta=1$. This is in contrast with Ref. [20], where $\beta=0.47$ is reported. The differences in exponents may be due to differences between our model and the specific realization of the random substrate model in Ref.[20]. This is supported by the fact that in nonlinear dynamical systems with quenched randomness, universality is often weaker than in equilibrium critical phenomena, so that some critical exponents may depend on details on short length scales. A striking demonstration of this for a directed polymer in a random medium, a problem closely related to the one considered here, was published recently [21].

free motion regime: $E>E_{2}$;

In this regime all particles possess a finite velocity at all times (See Fig. 3, inset). The instantaneous velocity of the center of mass is made up of a constant part plus a random washboard like modulation induced by motion over a fluctuating pinning landscape. In the limit: $E>>E_{2}$, the velocity tends to that of the analogous random network Eq. (3), and the relative fluctuating component of the velocity diminishes (Fig. 3). The drag force $F(U)$ approaches $E_{c}$.

Our model assumes the presence of local friction forces randomly distributed along the interface, while their microscopic origin are outside its scope. The stick-slip dynamics in the random substrate model is a consequence of the random arrangement of simple local interactions together with the cooperative effect of the elastic forces. While the decrease of macroscopic kinetic friction with velocity in real materials may have various causes, comparison with our results suggests surface inhomogeneity or randomness may play a significant role. 


\section{Scaling Properties of the Pinned Sate}

For both models, interfaces evolving by the dynamics described in Eq (1) or Eq (2), starting from a flat initial configuration, $\left(\xi_{i}=0\right.$ at $\left.\mathrm{t}=0\right)$ and subject to a constant drive $E$ below the pinning threshold, eventually reach a static state of strongly strained domains, or strain avalanches [22], seperated by virgin particles which never moved. For $E$ approaching $E_{c}$ from below, the random network model undergoes a second order dynamical phase transition $[9,10]$. The following numerical results show that the random substrate model follows a qualitatively similar critical behavior, but of a different universality class.

Fig. 2 shows a typical pinned interface for the random substrate model. Here interfaces are hindered by the tilted pinning lines, leading to characteristic triangular structures with a roughness exponent $\zeta=1$. In contrast, in the random network model, distortion of domains is much more pronounced and the roughness exponent is $\zeta=3 / 2[9,10]$. Similar to the random network model and to many other non-equilibrium systems (e.g. the scaling of avalanche sizes in self-organized criticality: [23]), the number of domains of size $\ell$ per unit length in the random substrate model has the generic scaling form:

$$
n(\ell) \sim \lambda^{\sigma} x^{-\tau} \Phi(x)
$$

where $x=\ell / \lambda(\epsilon)$, and $\Phi(x)$ is a slowly varying function for $x<1$ with a steep cutoff at $x>1$. The typical size of the largest domains, $\lambda$, diverges as $\epsilon^{-\nu}$ where $\epsilon=E-E_{1}$. The number of domains is not fixed. Close to the depinning threshold the number of virgin particles vanishes, and the whole system is tiled by strained domains. This global condition implies that the first moment of $n(l)$ is a slowly varying function of $\epsilon$ that does not become singular at criticality, leading to $\sigma=2$ for $\tau>1$, while for $\tau<1, \sigma=1-\tau$. The exponents of the random network model are different: $\tau=3 / 2, \sigma=2$, $\nu=2[9]$.

We obtained $n(\ell)$ for the random substrate model by solving Eq (1) numerically for an ensemble of 40 random realizations of chains of $10^{4}$ particles. Fig. 4 shows the rescaled histograms. The collapse of the data for different values of $\epsilon$ was achieved by rescaling using $\nu=2$ and $\tau=0.9 \pm 0.1$.

The analysis of higher moments of $n(\ell)$ does not show significant deviations from single parameter scaling. The values of critical exponents are siginificantly different than those of the random network model, confirming that the two models belong to distinct universality classes. 


\section{The Phase Diagram}

The properties of the random substrate model depend on the network's elastic stiffness. Intuition suggests that when the network becomes very rigid, the differences between the two systems should vanish. Fig. 5 represents a $(\kappa E)$ phase diagram, obtained from numerical studies of chains of $\mathrm{N}=100$. As the stiffness increases, the random substrate threshold $E_{1}$ approaches $E_{c}$. The second threshold $E_{2}$ is of the order of $V_{\max }$, the upper bound of the pinning strength distribution, and depends only weakly on $\kappa$.

Within the pinned regimes of both models, in region I of the phase diagram, configurations of strained domains of both models are roughly the same. The dark circles denotes values of $\kappa, E$ where the relative Hamming distance $D$ [24] between locations of strained domains for pairs of systems from each model with the same set of pinning strengths $\left\{V_{i}\right\}$, is smaller than 0.06 .

For the random network model the largest displacement $\xi_{\text {max }}(\epsilon)$ is bounded by $\lambda(\epsilon)^{3 / 2} \sim \epsilon^{-3}$. The scaling of the random substrate model with stiffness given by $\mathrm{Eq}(3)$, implies that for large $\kappa>\xi_{\max }$ the two models track. This is a sufficient condition, so it yields a lower bound for the boundary of region I where the two models coincide, given by values of $\kappa, E$ :

$$
E_{c}-E \sim \frac{1}{\kappa^{1 / 3}}
$$

An upper bound for $E(\kappa)$ is given by the relation: $E_{c}-E \sim 1 / \kappa[19]$. The corresponding boundary is also shown in Fig. 5. The fact that for stiff networks the two models are roughly equivalent, means that aspects of friction between very stiff solid bodies which are related to inhomogeneities on the interfaces can be described by the random network model, or by models based on it, elaborated to better conform to real solids.

\section{References}

[1] Charge Density Waves in Solids, edited by L. P. Gorkov and G. Gruner (Elsvier, Amsterdam, 1989) and references therein.

[2] H. Fukuyama, and P.A.Lee, Phys.Rev. B 17, 535 (1977); P. A. Lee and T. M. Rice, Phys. Rev. B19, 3970 (1970).

[3] M. Fiegelman, V. Geshkenbein, A. Larkin, and V. Vinokur, Phys. Rev. Lett. $63,2303,1989$.

[4] R. Burridge and L. Knopoff, Bull. Seis. Soc. Am. 57,341,1967. 
[5] J. M. Carlson and J. S. Langer, Rev. Mod. Phys. 66,658,1994, and references therein.

[6] D. S. Fisher, K. Dahmen, S. Ramanathan and Y. Ben-Zion, Phys. Rev. Lett., $78,4885,1997$.

[7] D. P. Villete and J. P. Golub, Phys. Rev. E 47,820,1993.

[8] D. S. Fisher, Phys. Rev. Lett. 50,1486, (1985); D. S. Fisher, Phys.Rev.B31, 1396 (1985).

[9] I. Webman, Phil. Magazine, B56743,(1987); I. Webman, (unpublished preprint, 1987).

[10] I.Webman in : Nonlinearities in Complex Systems Ed: S.Puri and S. Dattagupta, Narosa, New Delhi, 1997.

[11] A. A. Middleton and Daniel S. Fisher, Phys. Rev B 47, 3530, (1993); O. Narayan and A. A. Middleton, Phys. Rev. B49, 244,1994; O. Narayan and D. S. Fisher, Phys. Rev. Lett. 68,3615,1992; Phys. Rev. B46,11520, 1992.

[12] C. R. Myers and J.P. Sethna, Phys. Rev. B 47,11171,1993.

[13] G. Parisi, Europhys. Lett. 17, 673 (1992); G.Parisi and L. Pietronero, Europhysics Letters, 16, 321 (1991).

[14] , T. Giamarchi and P. Le Doussal, Phys. Rev. Lett. 72,1530,1994; Phys. Rev. Lett. $74,606,1995$.

[15] O. Pla and F. Nori, Phys. Rev. Lett. 67,919,1991.

[16] J. Krug, Phys. Rev. Lett. 75,1795,1995.

[17] L. Balents and M.P.A Fisher, Phys. Rev. Lett, 75,4270,1995.

[18] In the FLR-Fisher equation is similar to $\operatorname{Eq}(2)$, except that there $V(i)$ is multiplied by a cosine function of $\xi$. Using the ratchet condition is similar to replacing the cosine force by a derivative of a sawtooth potential, except that local backword motions down the slope of the sawtooth are excluded. These motions do not affect the large scale dynamics since the model has no inertia. The critical behavior of the random network model below threshold is in the same universality class as the CDW models of Fukuyama Lee and Rice and Fisher $[2,8]$, but can be solved analytically.

[19] I. Webman (unpublished)

[20] D. Cule and T. Hwa, Phys. Rev. Lett. 77, 278,1996; T. Hwa and D. Cule, Phys. Rev. Lett. 79, 4930,1997.

[21] Paolo De Los Rios and Yi-Cheng Zhang, Phys. Rev. Lett. 81,1023,1998.

[22] The equations of motion $\mathrm{Eq}(1), \mathrm{Eq}(2)$ have inifinitely many static solution. We focus on pinned states reached first from flat initial configurations in the presence of time independent external field. 
[23] P. Bak, C. Tang and K. Weisenfeld, Phys. Rev. Lett. 59,381,1987.

[24] The relevant Hamming distance is: $D=\frac{2 \sum\left\{\theta^{R S}\left(\xi_{i}\right)-\theta^{R N}\left(\xi_{i}\right)\right.}{\sum\left\{\theta^{R S}\left(\xi_{i}\right)+\theta^{R N}\left(\xi_{i}\right)\right.}$ where $\theta$ is a unit step function. 


\section{Random network model}

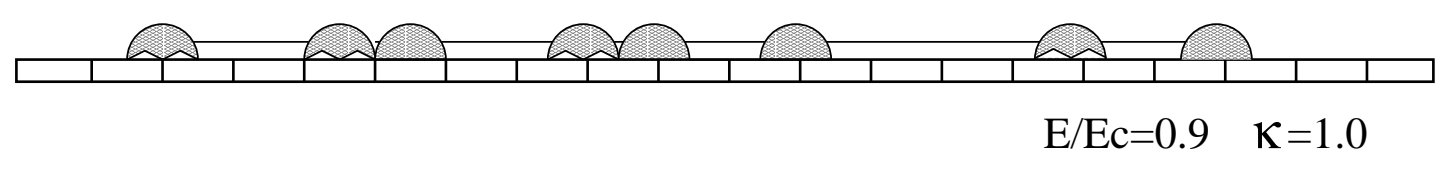

\section{Random substrate model}

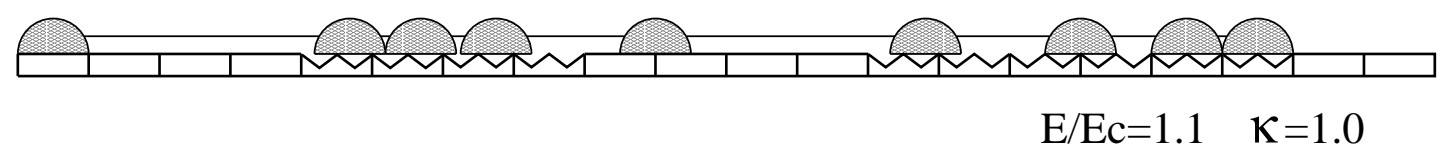

Fig. 1. Illustration of the random network and the random substrate models.

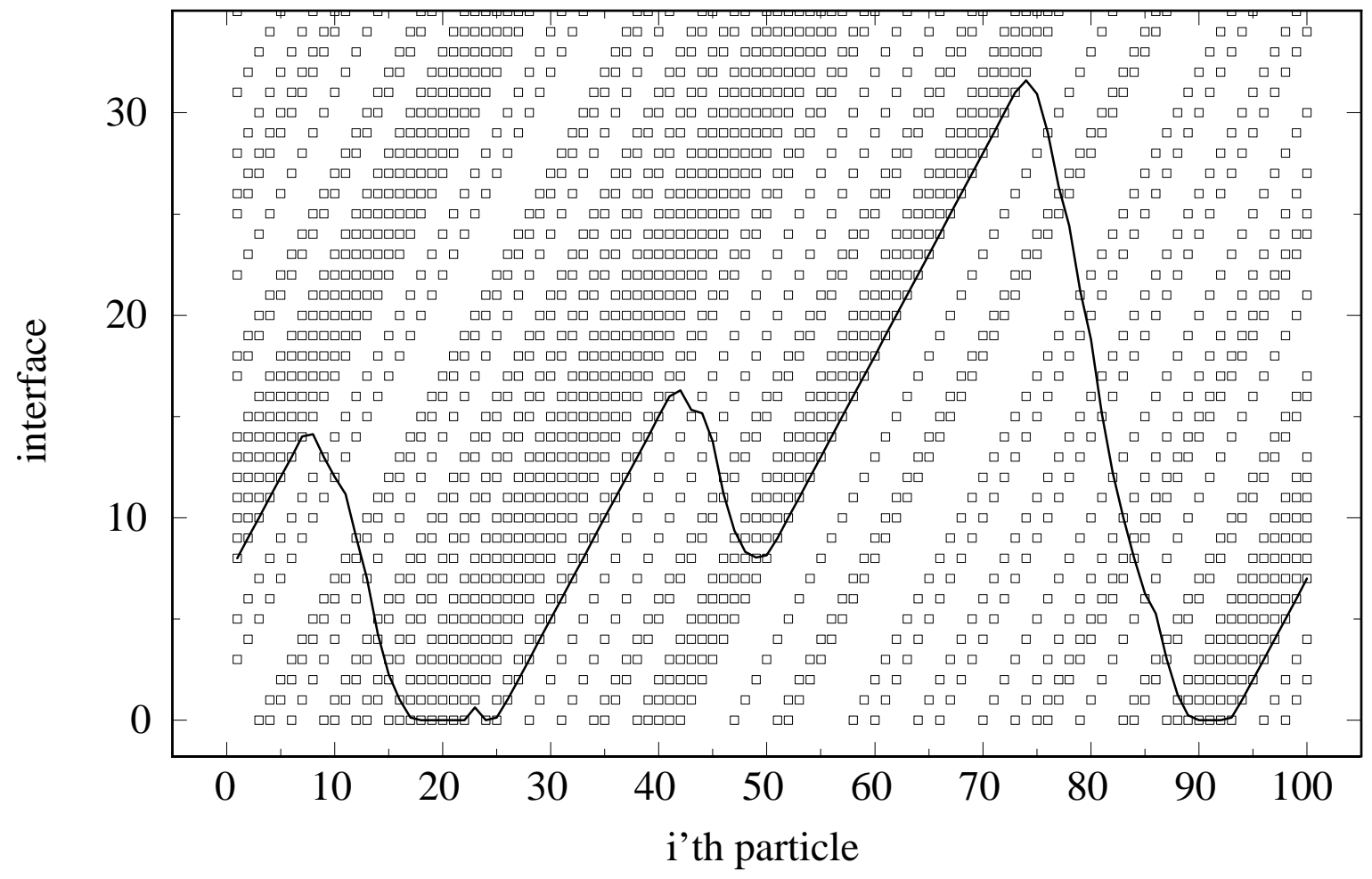

Fig. 2. A charactersitic pinned configuration in the interface representation of the random substrate model. 


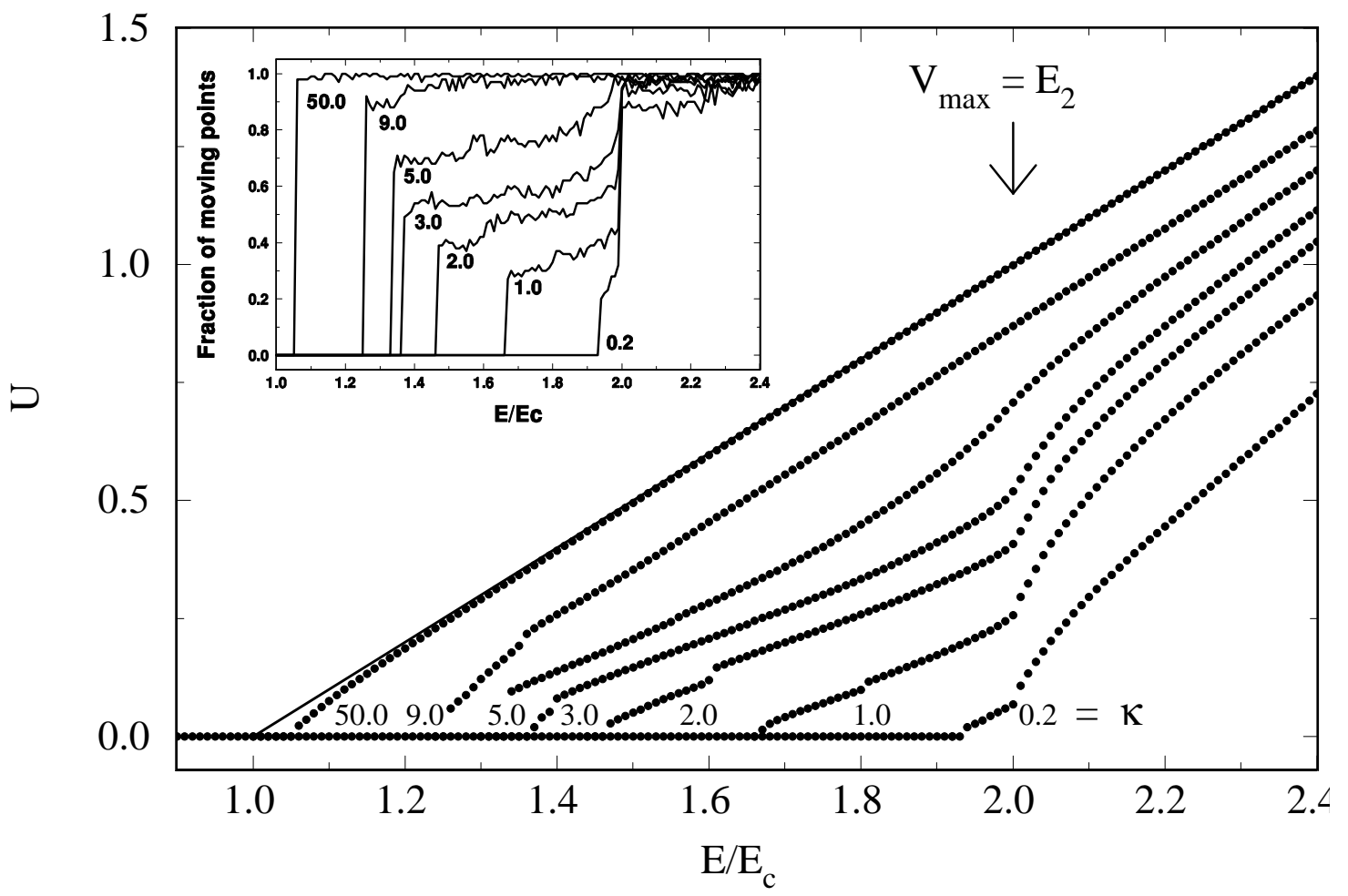

Fig. 3. Time averaged center of mass velocity vs. driving force for networks of various stiffness. Inset: Mean fraction of particles in motion.

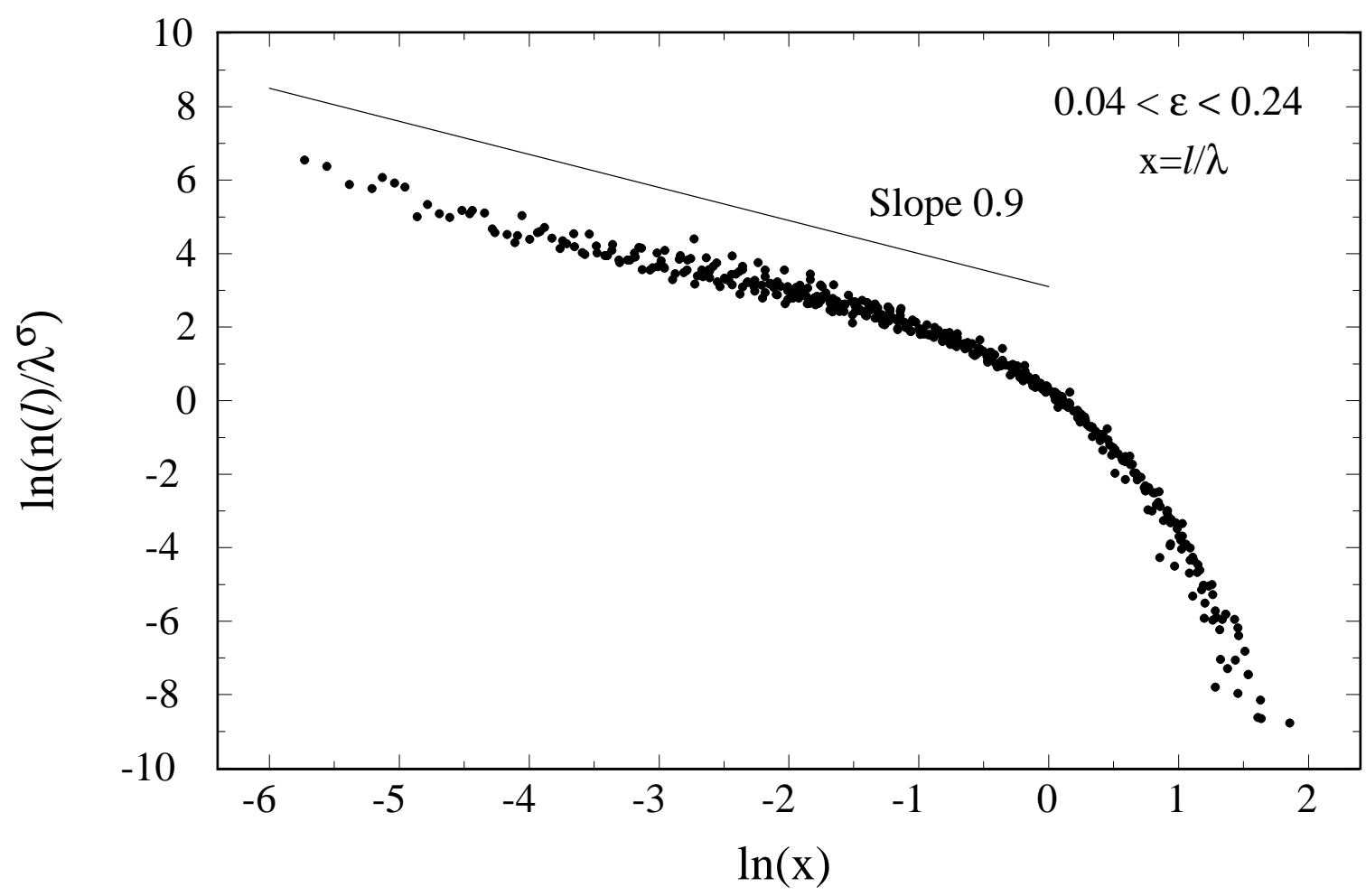

Fig. 4. Log-log plot of scaled distribution of sizes of strain avalanches in the pinned regime. 


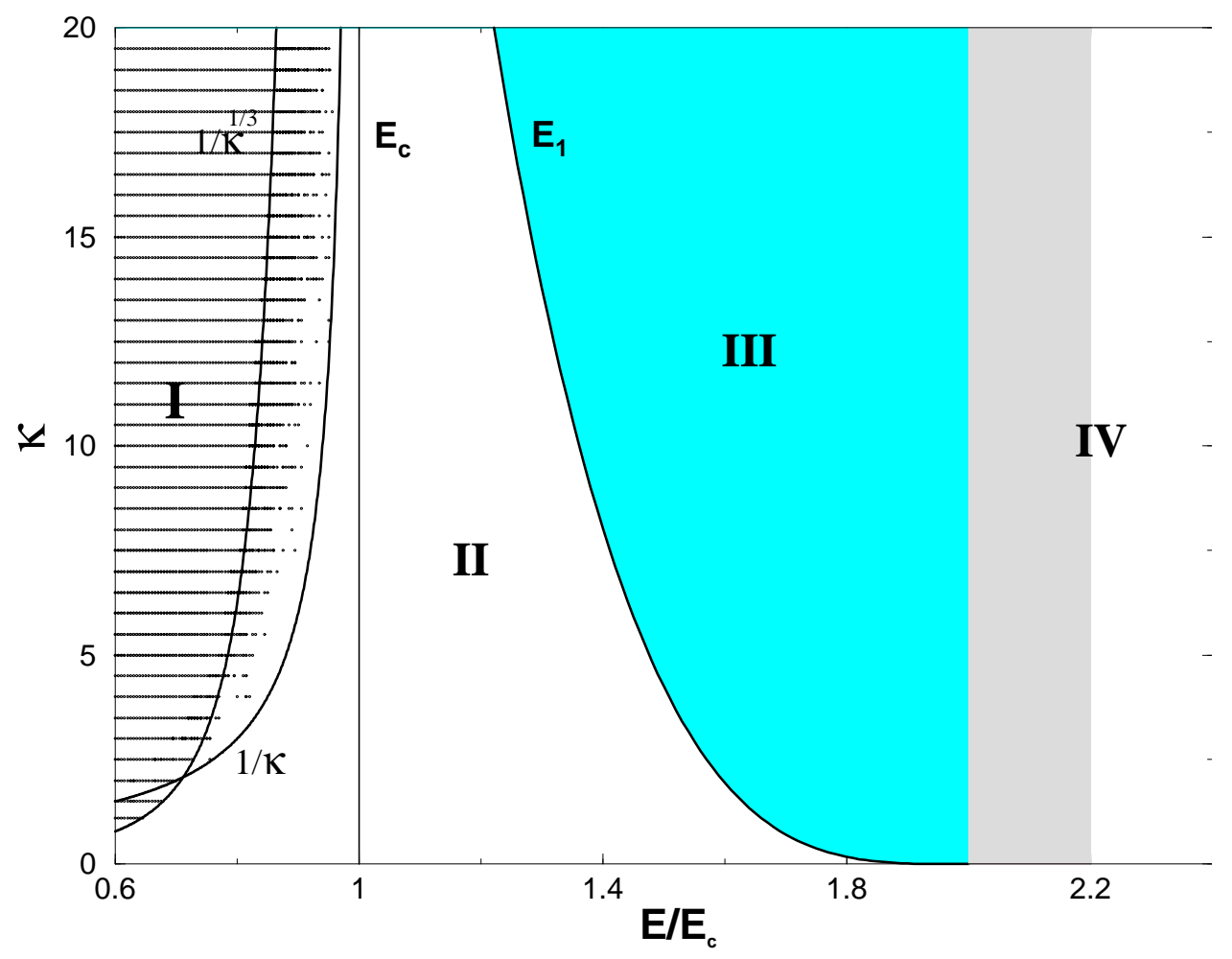

Fig. 5. Phase diagram in the $(\kappa, E)$. The network configurations are pinned in phase I and phase II. Phase III corresponds to stick-slip dynamics, and phase IV to free motion. In phase I the random network and the random substrate models yield similar pinned configurations. Theoretical lower and upper bounds of the boundary of phase I are shown. 